Pacific Journal of Mathematics

DIFFERENTIAL SIMPLICITY AND COMPLETE INTEGRAL 


\title{
DIFFERENTIAL SIMPLICITY AND COMPLETE INTEGRAL CLOSURE
}

\author{
YVES LeqUAIN
}

\begin{abstract}
Let $R$ be an integral domain containing the rational numbers, and let $R^{\prime}$ denote the complete integral closure of $R$. It is shown that if $R$ is differentiably simple, then $R$ need not be equal to $R^{\prime}$, even when $R$ is Noetherian, and then the relationship between $R$ and $R^{\prime}$ is studied.
\end{abstract}

Let $\mathscr{D}$ be any set of derivations of $R$. Seidenberg has shown that the conductor $C=\left\{x \in R \mid x R^{\prime} \subset R\right\}$ is a $\mathscr{D}$-ideal of $R$, so that when $R$ is $\mathscr{D}$-simple and $C \neq 0$, then $R=R^{\prime}$. We investigate here the situation when $C=0$.

The first observation that one must make is that it is no longer true that $R=R^{\prime}$ when $R$ is differentiably simple, even when $R$ is Noetherian. We show this in Example 2.2 where we construct a 1dimensional local domain containing the rational numbers which is differentiably simple but not integrally closed. This counterexamples a conjecture of Posner [4, p. 1421] and also answers affirmatively a question of Vasconcelos [6, p. 230].

Thus, it is not a redundant task to study the relationship between a differentiably simple ring $R$ and its complete integral closure. An important tool in this study is the technique of $\S 3$ which associates to any prime ideal $P$ of $R$ containing no $D$-ideal a rank-1, discrete valuation ring centered on $P$; by means of this, we show in Theorem 3.2 that over such a prime ideal $P$ of $R$ there lies a unique prime ideal of $R^{\prime}$. When $R$ is a Noetherian $\mathscr{D}$-simple ring with $\left\{P_{\alpha}\right\}_{\alpha \in A}$ as set of minimal prime ideals, Theorem 3.3 asserts that $R^{\prime}=\bigcap_{\alpha \in A}\left\{R_{\alpha} \mid R_{\alpha}\right.$ is the valuation ring associated with the minimal prime ideal $P_{\alpha}$; Corollary 3.5 asserts that $R^{\prime}$ is the largest $\mathscr{D}$-simple overring of $R$ having a prime ideal lying over every minimal prime ideal of $R$.

1. Preliminaries. Our notation and terminology adhere to that of Zariski-Samuel [7] and [8]. Throughout the paper we use $R$ to denote a commutative ring with $1, K$ to denote the total quotient ring of $R$, and $A$ to denote an ideal of $R ; A$ is proper if $A \neq R$. A derivation $D$ of $R$ is a map of $R$ into $R$ such that

$$
D(a+b)=D(a)+D(b) \text { and } D(a b)=a D(b)+b D(a)
$$

for all $a, b \in R$.

Such a derivation can be uniquely extended to $K$, and we shall 
also denote the extended derivation by $D . \quad D$ is said to be regular on a subring $S$ of $K$ if $D(S) \subset S$. If $\mathscr{D}$ is a family of derivations of $R, A$ is called a $\mathscr{D}$-ideal if $D(A) \subset A$ for every $D \in \mathscr{D}$; when $\mathscr{D}=$ $\{D\}$, we merely say $D$-ideal. If $R$ has no $\mathscr{D}$-ideal different from (0) and (1), $R$ is said to be $\mathscr{D}$-simple. We use $D^{(0)}(x)$ to denote $x$, and for $n \geqq 1 D^{(n)}(x)$ to denote $D\left(D^{(n-1)}(x)\right)$, i.e. the $n^{\text {th }}$ derivative of $x$; by induction one proves Leibnitz's rule:

$$
D^{(n)}(a b)=\sum_{i=0}^{n} C_{n}^{i} D^{(n-i)}(a) D^{(i)}(b) .
$$

We assume henceforth that $\mathscr{D}$ is a family of derivations of $R$ and that $D \in \mathscr{D}$. Let $\varphi: R \rightarrow S$ be a homomorphism onto; then

$$
D^{\prime}(\varphi(r))=\varphi(D(r))
$$

defines a derivation $D^{\prime}$ on $S$ if and only if the kernel $I$ of $\phi$ is a $D$ ideal. Suppose that $I$ is a $\mathscr{D}$-ideal, and write $\mathscr{D}^{\prime}$ to denote the set of derivations of $S$ thus induced by $\mathscr{D}$; if $A$ is a $\mathscr{D}$-ideal of $R$, then $\varphi(A)$ is a $\mathscr{D}^{\prime}$-ideal of $S$, and conversely if $B$ is a $\mathscr{D}^{\prime}$-ideal of $S$, then $\varphi^{-1}(B)$ is a $\mathscr{D}$-ideal of $R$ containing $I$. Thus, in particular, if $A$ is a maximal proper $\mathscr{D}$-ideal of $R$, then $R / A$ is $\mathscr{D}^{\prime}$-simple.

LEMMA 1.1. Let $D$ be a derivation of $R, M$ a multiplicative system of $R$, and $h: R \rightarrow R_{M}$ the canonical homomorphism. Then, we can define a derivation on $R_{M}$, which we also call $D$, by

$$
D\left(h(r)(h(m))^{-1}\right)=[h(m) h(D(r))-h(r) h(D(m))]\left(h\left(m^{2}\right)\right)^{-1} .
$$

Furthermore, if $A$ is a $D$-ideal of $R$, then $h(A) R_{M}$ is a D-ideal of $R_{M}$, and if $B$ is a $D$-ideal of $R_{M}$, then $h^{-1}(B)$ is a $D$-ideal of $R$.

Proof. $\operatorname{ker} h=\{x \in R \mid x m=0$ for some $m \in M\}$ is a $D$-ideal of $R$ since $0=D(x m)=x D(m)+m D(x)=x m D(m)+m^{2} D(x)=m^{2} D(x)$. Hence $D$ induces a derivation on $R / \operatorname{ker} h$, a derivation which can be then extended to $R_{M}$. The remainder of the lemma is straightforward.

Lemma 1.2. Let $\mathscr{D}$ be a family of derivations of $R$, and suppose that $R$ contains the rational numbers. Then, the radical of $a$. $\mathscr{D}$-ideal of $R$ is a $\mathscr{D}$-ideal.

Proof. See [2, Lemma 1.8, p. 12].

Corollary 1.3. If $P$ is a minimal prime divisor of a $\mathscr{D}$-ideal 
$A$, and $P$ does not contain an integer $\neq 0$, then $P$ is a $\mathscr{D}$-ideal.

Proof. Localize at $P$ and apply 1.1 and 1.2.

THEOREM 1.4. Let $A$ be a maximal proper $\mathscr{D}$-ideal of $R$, then

(i) $A$ is primary.

(ii) If $R / A$ has characteristic $p \neq 0$, then $\sqrt{A}$ is a maximal ideal.

(iii) If $R / A$ has characteristic 0 , then $A$ is prime.

Proof. (i) Suppose $x, y \in R, x \notin A$ and $x y \in A$; then, $\bigcup_{n=0}^{\infty}(A$ : $\left.y^{n}\right) \supset A: y>A$. But $\bigcup_{n=0}^{\infty}\left(A: y^{n}\right)$ is a $\mathscr{D}$-ideal; hence, by the maximality of $A, \bigcup_{n=0}^{\infty}\left(A: y^{n}\right)=R$ and there exists $n$ such that $y^{n} \in A$.

(ii) Let $P$ be a maximal ideal of $R$ containing $A$. Consider the ideal $B=\left(A,\left\{x^{p} \mid x \in P\right\}\right) \subset P$; since $R / A$ has characteristic $p, B$ is a $\mathscr{D}$-ideal; hence, by the maximality of $A, B=A$ and $P=\sqrt{A}$.

(iii) Since $R / A$ has characteristic $0, A$ contains no integer other than 0 , hence the prime ideal $P=\sqrt{A}$ contains no integer either, and by $1.3 P$ is a $\mathscr{D}$-ideal. Then, by the maximality of $A, P=A$.

COROLlaRY 1.5. Let $R$ be of characteristic 0. Then $R$ is $\mathscr{D}$ simple if $R$ contains the rational numbers and has no prime $\mathscr{D}$ ideal different from (0) and (1). If $R$ is $\mathscr{D}$-simple, then $R$ is a domain.

One should note that a $\mathscr{D}$-simple ring $R$ always contains a field, namely $F=\{x \in R \mid D(x)=0$ for all $D \in \mathscr{D}\}$; moreover, if the characteristic of $R$ is $p \neq 0,1.4$ shows that $R$ is a primary ring and hence is equal to its total quotient ring; so this case will not be of interest in our further considerations, and throughout the remainder of this section we shall be dealing with a $\mathscr{D}$-simple ring of characteristic 0 , which is then a domain containing the rational numbers.

Definition 1.6. Let $R$ be a domain with quotient field $K$. An element $x \in K$ is said to be quasi-integral over $R$ if there exists an element $d \in R, d \neq 0$, such that $d x^{n} \in R$ for all $n \geqq 1$. The set $R^{\prime}$ of all elements of $K$ that are quasi-integral over $R$ is a ring, called the complete integral closure of $R . R$ is said to be completely integrally closed if $R=R^{\prime}$. Note that if $R$ is Noetherian, the concepts of integral dependence and quasi-integral dependence over $R$ for elements of $K$ become the same.

LEMMA 1.7. Let $R$ be a domain with quotient field $K, S$ a ring 
such that $R \subset S \subset K$, and $\mathscr{D}$ a family of derivations of $R$ regular on $S$. Then $S$ is $\mathscr{D}$-simple if $R$ is $\mathscr{D}$-simple.

Proof. If $B$ is any $\mathscr{D}$-ideal of $S$, then $B \cap R$ is a $\mathscr{D}$-ideal of $R$, and if $B$ is different from (0) then $B \cap R$ is also different from (0) since $S \subset K$.

THEOREM 1.8. Let $R$ be a domain of characteristic 0 and $R^{\prime}$ its complete integral closure. Then $R^{\prime}$ is $\mathscr{D}$-simple if $R$ is $\mathscr{D}$-simple.

Proof. By [5, p. 168], any $D \in \mathscr{D}$ is regular on $R^{\prime}$, hence the theorem follows from 1.7.

2. Example of a 1-dimensional local ring which is D-simple but not integrally closed. First, in this section, we modify an idea of Akizuki in [1] to construct some 1-dimensional local ring $R$ of arbitrary characteristic such that the integral closure $\bar{R}$ is not a finite $R$-module.

THEOREM 2.1. Let $k$ be a field of arbitrary characteristic, $Y$ an indeterminate over $k, \pi=a_{1} Y+a_{2} Y^{3}+\cdots+a_{r} Y^{2^{r}-1}+\cdots$ an element of $k[[Y]]$ which is transcendental over $k[Y]^{1}$. Set

$$
\theta_{1}=\pi Y^{-1}, \theta_{r}=\left(\theta_{r-1}-a_{r-1}\right) Y^{-2^{r-1}}
$$

for $r \geqq 2$ (alternatively $\theta_{r}=a_{r}+a_{r+1} Y^{2^{r}}+\cdots+a_{s} Y^{2^{s}-2^{r}}+\cdots$ ); for $r \geqq 1$, set

$$
t_{r}=\left(\theta_{r}-a_{r}\right)^{2} \text { and } \pi_{r}=\pi-\left(a_{1} Y+\cdots+a_{r} Y^{2^{r}-1}\right) .
$$

Set also $T=k\left[Y, \pi, t_{1}, t_{2}, \cdots, t_{r} \cdots\right]$ and $P=(Y, \pi) T$. Note that $T \subset k[[Y]]$ and that $P \subset Y k[[Y]]$. Then,

(i) For $r>1, t_{r-1}=Y^{2^{r}}\left(a_{r}^{2}+t_{r}\right)+2 a_{r} Y \pi_{r}$ and $P$ is a maximal ideal of $T$.

(ii) For $r \geqq 1, \pi_{r}^{2}=Y^{2^{r+1}-2} \operatorname{tr}$ and $k(Y, \pi)$ is the quotient field of $T$.

(iii) The ring $R=T_{P}$ is a 1-dimensional local domain.

(iv) The integral closure $\bar{R}$ of $R$ is not a finite $R$-module.

Proof. (i) For $r>1$, we have

$$
t_{r-1}=\left(\theta_{r-1}-a_{r-1}\right)^{2}=\left(Y^{2^{r-1}} \theta_{r}\right)^{2}=Y^{2^{r}}\left(a_{r}^{2}+t_{r}\right)+2 a_{r} Y^{2^{r}}\left(\theta_{r}-a_{r}\right) .
$$

But

$$
Y^{2^{r}}\left(\theta_{r}-a_{r}\right)=Y\left[\pi-\left(a_{1} Y+\cdots+a_{r} Y^{2^{r}-1}\right)\right]=Y \pi_{r},
$$

${ }^{1}$ Such an element exists; take for example $\pi=a_{1} Y+a_{2} Y^{3}+\cdots+a_{r} Y^{2^{r !-1}}+\cdots$ with $a_{r} \neq 0$ for every $\mathrm{r} \geqq 1$. 
hence $t_{r-1}=Y^{2 r}\left(a_{r}^{2}+t_{r}\right)+2 a_{r} Y \pi_{r}$. Since furthermore $P \subset Y k[[Y]]$, $1 \notin P$, and $P$ is a maximal ideal of $T$.

(ii)

$$
\begin{aligned}
\pi_{r} & =\pi-\left(a_{1} Y+\cdots+a_{r} Y^{2^{r}-1}\right) \\
& =Y^{2 r-1}\left(a_{r+1} Y^{2 r}+\cdots+a_{r+\ell} Y^{2 r+\ell_{-2} r}+\cdots\right) \\
& =Y^{2 r-1}\left(\theta_{r}-a_{r}\right) ;
\end{aligned}
$$

thus $\pi_{r}^{2}=Y^{2^{r+1}-2} t_{r}$ and $k(Y, \pi)$ is the quotient field of $T$.

(iii) Let us show that $Y$ belongs to every nonzero prime ideal of $R$. Since $k(Y, \pi)$ is the quotient field of $R$ it suffices to show that $R\left[Y^{-1}\right]=k(Y, \pi)$. Let $\beta \in k[Y, \pi]$; then $\beta=\sum_{i=0}^{n} s_{i} \pi^{i}$ with $s_{i} \in k[Y]$. For any integer $r \geqq 1$, set $f_{r}=\sum_{i=0}^{n} s_{i}\left(a_{1} Y+\cdots+a_{r} Y^{2^{r}-1}\right)^{i}$; then

$$
f_{r+1}=\sum_{i=0}^{n} s_{i}\left(a_{1} Y+\cdots+a_{r} Y^{2^{r}-1}+a_{r+1} Y^{2^{r+1}-1}\right)^{i}=f_{r}+Y^{2^{r+1}-1} h_{r+1}
$$

with $h_{r+1} \in k[Y]$, and since $2^{r+1}-1>r$, we have $f_{r}=b_{0}+b_{1} Y+\cdots$ $+b_{r} Y^{r}+Y^{r+1} g_{r}$ and

$$
f_{r+1}=b_{0}+b_{1} Y+\cdots+b_{r} Y^{r}+b_{r+1} Y^{r+1}+Y^{r+2} g_{r+1}
$$

with $b_{0}, \cdots, b_{r}, b_{r+1} \in k$ and $g_{r}, g_{r+1} \in k[Y]$. Now, since

$$
\pi=\pi_{r}+\left(a_{1} Y+\cdots+a_{r} Y^{2 r-1}\right), \quad \beta=\sum_{i=0}^{n} s_{i} \pi^{i}=\pi_{r} \delta_{r}+f_{r}
$$

with $\delta_{r} \in T$. Hence, there exists $b_{0}, b_{1}, \cdots, b_{r}, \cdots \in k, \delta_{1}, \cdots, \delta_{r}, \cdots \in T$ and $g_{1}, \cdots, g_{r}, \cdots \in k[Y]$ such that

$$
\beta=\sum_{j=0}^{r} b_{j} Y^{j}+\pi_{r} \delta_{r}+Y^{r+1} g_{r} .
$$

Note that $\pi_{r} \in P$ and therefore that $\pi_{r}$ is a nonunit in $R$.

If $b_{0} \neq 0$, with $r=1$, the relation $\left(^{*}\right)$ gives that $\beta=b_{0}+\left(b_{1} Y+\right.$ $\left.\pi_{1} \delta_{1}+Y^{2} g_{1}\right)$ is a unit in $R$ and thus that $\beta^{-1} \in R \subset R\left[Y^{-1}\right]$.

If $b_{0}=b_{1}=\cdots=b_{r-1}=0$ and $b_{r} \neq 0$, the relation $\left(^{*}\right)$ gives $\beta=$ $Y^{r}\left(b_{r}+Y g_{r}\right)+\pi_{r} \delta_{r}$ where $w_{r}=b_{r}+Y g_{r}$ is a unit in $R$; then

$$
\beta\left(Y^{r} w_{r}-\pi_{r} \delta_{r}\right)=Y^{2 r} w_{r}^{2}-\pi_{r}^{2} \delta_{r}^{2}=Y^{2 r}\left(w_{r}^{2}-Y^{2^{r+1}-2 r-2} t_{r} \delta_{r}^{2}\right)
$$

where $w_{r}^{2}-Y^{2^{r+1}-2 r-2} t_{r} \delta_{r}^{2}$ is a unit in $R$, so that $\beta^{-1} \in R\left[Y^{-1}\right]$.

If $b_{r}=0$ for every $r \geqq 0$, then by the relation $\left(^{*}\right)$ we have

$$
\beta \in \bigcap_{r=1}^{\infty}\left(\pi_{r}, Y^{r+1}\right) T \subset \bigcap_{r=1}^{\infty} Y^{r+1} k[[Y]]=(0) .
$$

Thus, if $\beta \in k[Y, \pi]$, either $\beta^{-1} \in R\left[Y^{-1}\right]$ or $\beta=0$. If $\eta \in k(Y, \pi)$, then $\eta=\nu \lambda^{-1}$ with $\nu, \lambda \in k[Y, \pi], \lambda \neq 0$, so that $\eta \in R\left[Y^{-1}\right]$; hence $R\left[Y^{-1}\right]=k(Y, \pi)$. 
Now,

$$
\pi^{2}=\left(Y \theta_{1}\right)^{2}=\left[a_{1} Y+\left(\theta_{1}-a_{1}\right) Y\right]^{2}=\left(t_{1}-a_{1}^{2}\right) Y^{2}+2 a_{1} Y \pi
$$

so that $Y^{-1} \in R\left[\pi^{-1}\right], k(Y, \pi)=R\left[Y^{-1}\right] \subset R\left[\pi^{-1}\right]$, and $\pi$ belongs also to every nonzero prime ideal of $R$. Thus $P R=(Y, \pi) R$, which is the unique maximal ideal of $R$ and which is contained in every nonzero prime ideal of $R$, is the only nonzero prime ideal of $R$. As furthermore $P R$ is finitely generated, $R$ is a 1 -dimensional local ring.

(iv) First, let us show that $\theta_{1}=\pi Y^{-1} \notin T$. Suppose that $\theta_{1} \in T=$ $k\left[Y, \pi, t_{1}, \cdots, t_{r}, \cdots\right]$; then $\theta_{1}=f\left(\pi, t_{1}, \cdots, t_{\ell}\right)$ where $f$ is a polynomial in $\ell+1$ indeterminates over $k[Y]$. For $r<\ell$, by (i), $t_{r}$ can be expressed as a linear combination of $1, t_{\ell}$ and $\pi$ with coefficients in $k[Y]$, hence $\theta_{1}=f\left(\pi, t_{1}, \cdots, t_{\ell}\right)=F\left(\pi, t_{\ell}\right)=F\left(Y \theta_{1},\left(\theta_{\ell}-a_{\ell}\right)^{2}\right)$ where $F$ is a polynomial in two indeterminates over $k[Y]$. Furthermore, by definition $\theta_{r-1}=Y^{2^{r-1}} \theta_{r}+a_{r-1}$, hence $\theta_{1}=Y^{2} \iota_{-2} \theta_{\ell}+\beta_{\ell}$ with $\beta_{\ell} \in k[Y]$ and we have

$$
Y^{2^{\ell-2}} \theta_{\ell}=G\left(Y^{2 \ell-1} \theta_{\ell},\left(\theta_{\ell}-a_{\ell}\right)^{2}\right)
$$

where $G$ is a polynomial in two indeterminates over $k[Y]$; but $\pi$ being transcendental over $k[Y], \theta_{\ell}$ is transcendental over $k[Y]$ also, and the relation (**) has to be an identity, which is absurd. Thus, $\theta_{1} \notin T$.

Now, let $R^{*}$ be the completion of $R$ with the $(P R)$-adic topology; $\left\{\pi_{r}\right\}_{r \geqq 0}$ is a Cauchy sequence in $R$. Suppose that $\pi_{r} \in P^{2} R$ for some $r \geqq 1$; since $P^{2}$ is a primary ideal of $T$, we have $\pi_{r} \in P^{2} R \cap T=P^{2} \subset Y T$, and $\pi=\pi_{r}+\left(a_{1} Y+\cdots+a_{r} Y^{2^{r}-1}\right) \in Y T$ which is absurd since $\theta_{1} \notin T$. Thus, for every $r \geqq 0, \pi_{r} \notin P^{2} R$ and $\beta=\lim _{r} \pi_{r}$ is $\neq 0$. However, we also have $\beta^{2}=\lim _{r} \pi_{r}^{2}=\lim _{r} Y^{2^{r+1}-2} t_{r}=0$; hence $R^{*}$ has a nonzero nilpotent element and $\bar{R}$ is not a finite $R$-module [1, p. 330].

EXAMPLE 2.2. Let $Q$ be the rational numbers, $\left(X_{1}, \cdots, X_{r}, \cdots\right)$ a set of indeterminates over $Q$ and $k=Q\left(X_{1}, \cdots, X_{r}, \cdots\right)$. Let

$$
\pi=b_{1} X_{1} Y+\cdots+b_{r} X_{r} Y^{2 r-1}+\cdots
$$

be transcendental over $k[Y]$ with $b_{i} \in Q-\{0\}$ for every $i \geqq 1^{2}$. Construct the rings $T=k\left[Y, \pi, t_{1}, \cdots, t_{r}, \cdots\right]$ and $R=T_{P}$ as in 2.1. On the quotient field $k(Y, \pi)=Q\left(X_{1}, \cdots, X_{r}, \cdots ; Y, \pi\right)$ define a derivation $D$ by

$$
\begin{aligned}
D(q) & =0 \quad \text { for every } \quad q \in Q \\
D(Y) & =1 \\
D(\pi) & =3 b_{2} X_{2} Y^{2}+b_{1} X_{1} \\
D\left(X_{1}\right) & =0
\end{aligned}
$$

2 There exists such a $\pi$ since $k$ is countable. 


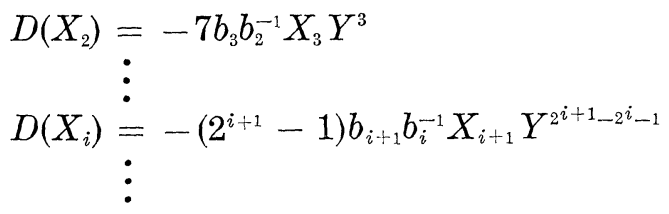

Then,

(i) $D$ is regular on $R$

(ii) $R$ is a 1 -dimensional local $D$-simple ring which is not integrally closed.

Proof. (i) Since $R=T_{P}$, it suffices to show that $D(T) \subset R$. By definition of $D$ we already have $D(k) \subset R, D(Y) \in R$ and $D(\pi) \in R$; hence it remains to show that $D\left(t_{r}\right) \in R$ for every $r \geqq 1$. Differentiating $\pi_{r}^{2}=Y^{2^{r+1}-2} t_{r}$, we get $2 \pi_{r} D\left(\pi_{r}\right)=Y^{2^{r+1}-2} D\left(t_{r}\right)+\left(2^{r+1}-2\right) Y^{2^{r+1}-3} t_{r}$; but $t_{r} \in Y R$ by 2.1 , hence $D\left(t_{r}\right) \in R$ if and only if $\pi_{r} D\left(\pi_{r}\right) \in Y^{2^{r+1}-2} R$. Let us show that in fact we have $D\left(\pi_{r}\right) \in Y^{2^{r+1}-2} R$. From $\pi_{1}=\pi-$ $b_{1} X_{1} Y$ we get $D\left(\pi_{1}\right)=D(\pi)-b_{1} X_{1}=3 b_{2} X_{2} Y^{2}$; by induction, if we suppose that $D\left(\pi_{r-1}\right)=\left(2^{r}-1\right) b_{r} X_{r} Y^{2^{r-2}}$ and if we differentiate the relation $\pi_{r}=\pi_{r-1}-b_{r} X_{r} Y^{2^{r}-1}$, we get $D\left(\pi_{r}\right)=\left(2^{r+1}-1\right) b_{r+1} X_{r+1} Y^{2^{r+1}-2} \epsilon$ $Y^{2^{r+1}-2} R$. Hence $D$ is regular on $R$.

(ii) The only prime ideal of $R$ which is not (0) or (1) is $P R=$ $(Y, \pi) R$; it is not a $D$-ideal since $D(Y)=1$; thus by $1.5, R$ is $D$-simple. Furthermore by 2.1. $R$ is a 1-dimensional local, not integrally closed, domain.

3. On the complete integral closure of a $\mathscr{D}$-simple ring. We have seen in the preliminaries that a $\mathscr{D}$-simple ring of characteristic $p \neq 0$ is equal to it total quotient ring. In this section we are concerned with rings of characteristic 0 . Henceforth, $R$ will denote a ring containing the integers.

THeOREM 3.1. Let $R$ be a ring, $D$ a derivation on $R, P$ a prime ideal of $R$ containing no D-ideal other than (0). Define $v: R \backslash\{0\} \rightarrow$ \{nonnegative integers\} by $v(x)=n$ if $D^{(i)}(x) \in P$ for $i=0, \cdots, n-1$ and $D^{(n}(x) \notin P$. Then,

(i) $R$ is domain.

(ii) $v$ is rank-1-discrete valuation whose valuation ring $R_{v}$ contains $R$ and whose maximal ideal $M_{v}$ lies over $P$.

(iii) $D$ is regular on $R_{v}$ and $R_{v}$ is D-simple.

Proof. (i) If $n$ is any integer, $D(n)=0$ and $n R$ is a $D$-ideal of $R$; hence 0 is the only integer contained in $P$. Now, $(0)$ is a $D$ ideal, hence by 1.3 any minimal prime divisor $Q$ of $(0)$ is a $D$-ideal also; then, by the hypothesis made on $P$, we have $(0)=Q$ and $R$ is a domain. 
(ii) Let $x$ and $y$ be two nonzero elements of $R$, and let $v(x)=n$, $v(y)=m, n \leqq m$. For every $i$ such that $0 \leqq i \leqq n-1$, both $D^{(i)}(x)$ and $D^{(i)}(y)$ belong to $P$, hence $D^{(i)}(x+y) \in P$ and

$$
v(x+y) \geqq n=\inf \{v(x), v(y)\} \text {. }
$$

Let $k$ be such that $0 \leqq k \leqq n+m-1$. For $0 \leqq i \leqq \inf \{k, n-1\}$ we have $D^{(i)}(x) \in P$, hence also $C_{k}^{i} D^{(i)}(x) D^{(k-i)}(y) \in P$; for $n \leqq k$ and $n \leqq i \leqq k$ we have $0 \leqq k-i \leqq k-n \leqq m-1$, hence $D^{(k-i)}(y) \in P$ and $C_{k}^{i} D^{(i)}(x) D^{(k-i)}(y) \in P$; thus

$$
D^{(k)}(x y)=\sum_{i=0}^{k} C_{k}^{i} D^{(i)}(x) D^{(k-i)}(y) \in P .
$$

Now,

$$
\begin{aligned}
D^{(n+m)}(x y)= & \sum_{i=0}^{n+m} C_{n+m}^{i} D^{(i)}(x) D^{(n+m-i)}(y) ; \sum_{i=0}^{n-1} C_{n+m}^{i} D^{(i)}(x) D^{(n+m-i)}(y) \\
& +\sum_{i=n+1}^{n+m} C_{n+m}^{i} D^{(i)}(x) D^{(n+m-1)}(y) \in P
\end{aligned}
$$

whereas $C_{n+m}^{n} D^{(n)}(x) D^{(m)}(y) \notin P$ since $C_{n+m}^{n}, D^{(n)}(x), D^{(m)}(y) \notin P$; thus

$$
D^{(n+m)}(x y) \notin P, \quad v(x y)=n+m=v(x)+v(y)
$$

and $v$ is a valuation, rank-1-discrete since its value group is the group of integers. Furthermore, we obviously have $R \subset R_{v}$ and $M_{v} \cap R=P$.

(iii) Let $a b^{-1}$ be any element of $R_{v}$ with $a, b \in R, b \neq 0, v(a) \geqq$ $v(b)$; then $D\left(a b^{-1}\right)=[b D(a)-a D(b)] b^{-2}$. If $v(a)>v(b)$, then $v(D(a))=$ $v(a)-1 \geqq v(b)$ and $v(D(b)) \geqq v(b)-1$ so that

$$
v(b D(a)-a D(b)) \geqq \inf \{v(b)+v(D(a)), v(a)+v(D(b))\} \geqq 2 v(b)
$$

and $D\left(a b^{-1}\right) \in R_{v}$. If $v(a)=v(b)=0$, then $v(b D(a)-a D(b)) \geqq 0=2 v(b)$ and $D\left(a b^{-1}\right) \in R_{v}$. If $v(a)=v(b)=n>0$, then $v(b D(a))=v(a D(b))=$ $2 n-1$, so that $D^{(k)}(b D(a)-a D(b)) \in P$ for every $k \leqq 2 n-2$; furthermore we have

$$
D^{(2 n-1)}(b D(a))=\sum_{i=0}^{2 n-1} C_{2 n-1}^{i} D^{(i)}(b) D^{(2 n-i)}(a)=\alpha_{1}+C_{2 n-1}^{n} D^{(n)}(b) D^{(n)}(a)
$$

with $\alpha_{1} \in P$, and similarly $D^{(2 n-1)}(a D(b))=\alpha_{2}+C_{2 n-1}^{n} D^{(n)}(a) D^{(n)}(b)$ with $\alpha_{2} \in P$, so that $D^{(2 n-1)}(b D(a)-a D(b))=\alpha_{1}-\alpha_{2} \in P$; hence, $v(b D(a)-$ $a D(b)) \geqq 2 n$ and $D\left(a b^{-1}\right) \in R_{v}$. Thus $D$ is regular on $R_{v}$. Moreover, $R_{v}$ is $D$-simple since if $A \neq(0)$ were a $D$-ideal of $R_{v}$, then $A \cap R \neq(0)$ would be a $D$-ideal of $R$ contained in $P$, which would be absurd.

THeOREM 3.2. Let $R$ be a domain with quotient field $K, S$ a ring such that $R \subset S \subset K$ and $D$ a derivation of $R$ regular on $S$. 
Let $P$ be a prime ideal of $R$ such that $R_{P}$ is $D$-simple. Then,

(i) There is at most one prime ideal $Q$ of $S$ lying over $P, Q$ being a minimal prime ideal when $P$ is.

(ii) If $S$ is the complete integral closure $R^{\prime}$ of $R$ there is exactly one prime ideal $P^{\prime}$ of $R^{\prime}$ lying over $P$.

Proof. (i ) Let $Q$ be a prime ideal of $S$ such that $Q \cap R=P$. Being regular on $S, D$ is also regular on $S_{Q}$, and $S_{Q}$ is $D$-simple since $S_{Q} \supset R_{P}$. Define $v: R \backslash\{0\} \rightarrow$ nnonnegative integers $\}$ by $v(x)=n$ if

$$
D^{(0)}(x), \cdots, D^{(n-1)}(x) \in P \quad \text { and } \quad D^{(n)}(x) \notin P,
$$

and $w: S \backslash\{0\} \rightarrow$ nonnegative integers $\}$ by

$$
w(y)=m \text { if } D^{(0)}(y), \cdots, D^{(m-1)}(y) \in Q
$$

and $D^{(m)}(y) \notin Q$. By 3.1, $v$ and $w$ extend to valuations of $K$; furthermore, for $x \in R$ we have $D^{(k)}(x) \in P$ if and only if $D^{(k)}(x) \in Q$ since $Q \cap R=P$; hence $v=w$, and $Q=M_{V} \cap S$ where $M_{V}$ is the maximal ideal of the valuation ring $R_{v}$ of $v$.

If $P$ is a minimal prime ideal of $R$, suppose that $Q^{\prime}$ is a prime ideal of $S$ such that $0<Q^{\prime} \subset Q$. We have $0<Q^{\prime} \cap R \subset Q \cap R=P$ and $Q^{\prime} \cap R=P$ by the minimality of $P$; then $Q^{\prime}=Q$ since $Q$ is the only prime ideal of $S$ lying over $P$.

(ii) By [5, p. 168] every derivation of $R$ is regular on $R^{\prime}$. Being a rank-1 valuation ring, $R_{v}$ is completely integrally closed and contains $R^{\prime}$. Then, $P^{\prime}=M_{Y} \cap R^{\prime}$ is a prime ideal of $R^{\prime}$ lying over $P$; of course, by (i), $P^{\prime}$ is unique.

THEOREM 3.3. Let $R$ be a Noetherian $\mathscr{D}$-simple ring and $\bar{R}$ its integral closure. Let $\left\{P_{\alpha}\right\}_{\alpha \in A}$ be the set all the minimal prime ideals of $R$. Then,

(i) For every $\alpha \in \Lambda$, there exists $D \in \mathscr{D}$ such that $R_{P_{\alpha}}$ is $D$ simple, and there exists a unique prime ideal $\bar{P}_{\alpha}$ of $\bar{R}$ lying over $P_{\alpha}$.

(ii) $\left\{\bar{P}_{\alpha}\right\}_{\alpha \in A}$ is the set of all the minimal prime ideals of $\bar{R}$.

(iii) Let $D \in \mathscr{D}$ such that $D\left(P_{\alpha}\right) \not \subset P_{\alpha}, w_{\alpha}$ the valuation associated by 3.1, and $R_{\alpha}$ its valuation ring. Then $R_{\alpha}=\bar{R}_{\bar{P}_{\alpha}}$ (hence, any two derivations $D$ and $D^{\prime}$ such that $D\left(P_{\alpha}\right) \not \subset P_{\alpha}$ and $D^{\prime}\left(P_{\alpha}\right) \not \subset P_{\alpha}$ give rise to the same valuation $w_{\alpha}$ ).

(iv) $\bar{R}=\bigcap_{\alpha \in \Lambda} R_{\alpha}$.

Proof. (i) Being $\mathscr{D}$-simple, $R$ is a domain containing the rational numbers, and for any $\alpha \in \Lambda$, there exists $D \in \mathscr{D}$ such that $D\left(P_{\alpha}\right) \not \subset P_{\alpha}$, and by $1.3, R_{P_{a}}$ is $D$-simple. Then, by 3.2 , there exists a unique prime ideal $\bar{P}_{\alpha}$ of $\bar{R}$ lying over $P_{\alpha}$. 
(ii) That every $P_{\alpha}$ is a minimal prime ideal of $\bar{R}$ is given by 3.2 . Now, let $\bar{P}$ be a minimal prime ideal of $\bar{R}$, and let $P=\bar{P} \cap R$; let $M$ be a minimal prime ideal of $R$ contained in $P$; by [3, (10.8), p. 30] there exists a prime ideal $\bar{M}$ of $\bar{R}$ lying over $M$; since $\bar{P}$ is the only prime ideal of $\bar{R}$ lying over $P$, we have $\bar{M} \subset \bar{P}$ by [3, (10.9), p. 30], hence $\bar{M}=\bar{P}$, and $P=\bar{P} \cap R=M$ is a minimal prime ideal of $R$.

(iii) Since $R$ is Noetherian, $\bar{R}$ is a Krull ring [3, (33.10), p. 118], and $\bar{R}_{\bar{P}_{\alpha}}$ is a rank-1-discrete valuation ring. As furthermore $\bar{R}_{\bar{P}_{\alpha}} \subset R_{\alpha}$ we get $\bar{R}_{\bar{P} \alpha}=R_{\alpha}$.

(iv) $\bar{R}$ is a Krull ring and $\left\{\bar{P}_{\alpha}\right\}_{\alpha \in A}$ is the set of all the minimal prime ideals of $\bar{R}$; thus $\bar{R}=\bigcap_{\alpha \in \Lambda} \bar{R}_{\bar{P}_{\alpha}}=\bigcap_{\alpha \in \Lambda} R_{\alpha}$.

CoRollary 3.4. Let $R$ be a Noetherian $\mathscr{D}$-simple ring with quotient field $K$. Let $S$ be a ring such that $R \subset S \subset K$ and such that every $D \in \mathscr{D}$ is regular on $S$. Then, the following statements are equivalent:

(i) For every minimal prime ideal $P$ of $R$ there exists a (unique) prime ideal $Q$ of $S$ lying over $P$.

(ii) $S$ is integral over $R$.

(iii) For every prime ideal $M$ of $R$ there exists a (unique) prime ideal $N$ of $S$ lying over $M$.

Proof. That (ii) $\Rightarrow$ (iii) is a consequence of $[3,(10.7)$, p. 30] and 3.2; that (iii) $\Rightarrow$ (i) is obvious. Now, let $\left\{P_{\alpha}\right\}_{\alpha \in A}$ be the set of the minimal prime ideals of $R,\left\{w_{\alpha}\right\}_{\alpha \in \Lambda}$ the associated valuations and $\left\{R_{\alpha}\right\}_{\alpha \in A}$ the valuation rings of the $w_{\alpha}$ 's. For any $\alpha \in \Lambda$, let $D \in \mathscr{D}$ be such that $D\left(P_{\alpha}\right) \not \subset P_{\alpha}$, and let $Q_{\alpha}$ be a prime ideal of $S$ lying over $P_{\alpha} ; S_{Q_{\alpha}}$ is $D$-simple, the valuation associated to $Q_{\alpha}$ is equal to $w_{\alpha}$ and $S \subset R_{\alpha}$. Hence, $S \subset \bar{R}=\bigcap_{\alpha \in A} R_{\alpha}$.

CoROLlary 3.5. Let $R$ be a Noetherian $\mathscr{D}$-simple ring with quotient field $K$, and $\bar{R}$ its integral closure. Then,

(i) $\bar{R}$ is the largest $\mathscr{D}$-simple overring of $R$ in $K$ having a prime ideal lying over every prime ideal of $R$.

(ii) $\bar{R}$ is the largest $\mathscr{D}$-simple overring of $R$ in $K$ having a prime ideal lying over every minimal prime ideal of $R$.

Proof. Apply 3.4.

The author wishes to acknowledge the many helpful discussions on the topics of this paper he had with Professor Ohm. 


\section{REFERENCES}

1. Y. Akizuki, Einige Bemerkungen uber primare Integritatsbereiche mit Teilerkettensatz, Proc. Physico-Math Soc. Japan 17 (1935), 327-336.

2. I. Kaplansky, An Introduction to Differential Algebra, Actualités Scientifiques et industrielles, Herman, 1957.

3. M. Nagata, Local Rings, John Wiley and Sons, New York, 1962.

4. E. C. Posner, Integral closure of rings of solutions of linear differential equations, Pacific J. Math. 12 (1962), 1417-1422.

5. A. Seidenberg, Derivations and integral closure, Pacific J. Math. 16 (1966), 167-173.

6. W. V. Vasconcelos, Derivations of commutative Noetherian rings, Math. Zeit 112 (1969), 229-233.

7. O. Zariski and P. Samuel, Commutative Algebra, V. 1, Van Nostrand Co., Princeton, 1958.

8. - Commutative Algebra, V. 2, Van Nostrand Co., Princeton, 1960.

Received February 9, 1970. This paper is part of the author's doctoral thesis written under the direction of Professor Jack Ohm at Louisiana State University.

LoUisiana State University

Baton Rouge, Louisiana

AND

Institute de Matematica Pura and Aplicada

RUa Luis de Camões 68

Rio de Janeiro 58, GB, Brazil 



\title{
PACIFIC JOURNAL OF MATHEMATICS
}

\section{EDITORS}

H. SAMELSON

Stanford University

Stanford, California 94305

C. R. HobвY

University of Washington

Seattle, Washington 98105

\section{J. DugunduI}

Department of Mathematics

University of Southern California

Los Angeles, California 90007

RICHARD ARENS

University of California

Los Angeles, California 90024

\section{ASSOCIATE EDITORS}
E. F. BECKENBACH
B. H. Neumann
F. WOLE
K. Yoshida

\section{SUPPORTING INSTITUTIONS}

\author{
UNIVERSITY OF BRITISH COLUMBIA \\ CALIFORNIA INSTITUTE OF TECHNOLOGY \\ UNIVERSITY OF CALIFORNIA \\ MONTANA STATE UNIVERSITY \\ UNIVERSITY OF NEVADA \\ NEW MEXICO STATE UNIVERSITY \\ OREGON STATE UNIVERSITY \\ UNIVERSITY OF OREGON \\ OSAKA UNIVERSITY \\ UNIVERSITY OF SOUTHERN CALIFORNIA
}

\author{
STANFORD UNIVERSITY \\ UNIVERSITY OF TOKYO \\ UNIVERSITY OF UTAH \\ WASHINGTON STATE UNIVERSITY \\ UNIVERSITY OF WASHINGTON

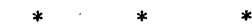 \\ AMERICAN MATHEMATICAL SOCIETY \\ CHEVRON RESEARCH CORPORATION \\ NAVAL WEAPONS CENTER
}

The Supporting Institutions listed above contribute to the cost of publication of this Journal, but they are not owners or publishers and have no responsibility for its content or policies.

Mathematical papers intended for publication in the Pacific Journal of Mathematics should be in typed form or offset-reproduced, (not dittoed), double spaced with large margins. Underline Greek letters in red, German in green, and script in blue. The first paragraph or two must be capable of being used separately as a synopsis of the entire paper. The editorial "we" must not be used in the synopsis, and items of the bibliography should not be cited there unless absolutely necessary, in which case they must be identified by author and Journal, rather than by item number. Manuscripts, in duplicate if possible, may be sent to any one of the four editors. Please classify according to the scheme of Math. Rev. Index to Vol. 39. All other communications to the editors should be addressed to the managing editor, Richard Arens, University of California, Los Angeles, California, 90024.

50 reprints are provided free for each article; additional copies may be obtained at cost in multiples of 50 .

The Pacific Journal of Mathematics is published monthly. Effective with Volume 16 the price per volume (3 numbers) is $\$ 8.00$; single issues, $\$ 3.00$. Special price for current issues to individual faculty members of supporting institutions and to individual members of the American Mathematical Society: $\$ 4.00$ per volume; single issues $\$ 1.50$. Back numbers are available.

Subscriptions, orders for back numbers, and changes of address should be sent to Pacific Journal of Mathematics, 103 Highland Boulevard, Berkeley, California, 94708.

PUBLISHED BY PACIFIC JOURNAL OF MATHEMATICS, A NON-PROFIT CORPORATION

Printed at Kokusai Bunken Insatsusha (International Academic Printing Co., Ltd.), 7-17, Fujimi 2-chome, Chiyoda-ku, Tokyo, Japan. 


\section{Pacific Journal of Mathematics}

\section{Vol. 36, No. $3 \quad$ BadMonth, 1971}

E. M. Alfsen and B. Hirsberg, On dominated extensions in linear subspaces of

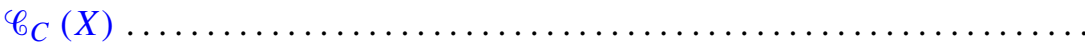

Joby Milo Anthony, Topologies for quotient fields of commutative integral

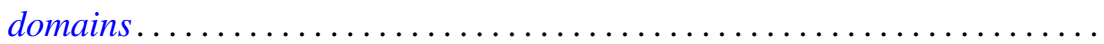

V. Balakrishnan, G. Sankaranarayanan and C. Suyambulingom, Ordered cycle lengths in a random permutation .......................... 603

Victor Allen Belfi, Nontangential homotopy equivalences............... 615

Jane Maxwell Day, Compact semigroups with square roots .............. 623

Norman Henry Eggert, Jr., Quasi regular groups of finite commutative nilpotent algebras .......................................... 631

Paul Erdős and Ernst Gabor Straus, Some number theoretic results ......... 635

George Rudolph Gordh, Jr., Monotone decompositions of irreducible Hausdorff continua .............................................

Darald Joe Hartfiel, The matrix equation $A X B=X \ldots \ldots \ldots \ldots \ldots \ldots . \ldots 69$

James Howard Hedlund, Expansive automorphisms of Banach spaces. II . . . . 671

I. Martin (Irving) Isaacs, The p-parts of character degrees in p-solvable

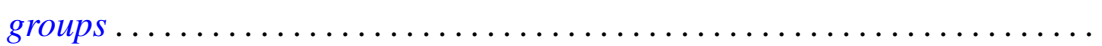

Donald Glen Johnson, Rings of quotients of $\Phi$-algebras ............... 693

Norman Lloyd Johnson, Transition planes constructed from semifield planes....

Anne Bramble Searle Koehler, Quasi-projective and quasi-injective modules.

James J. Kuzmanovich, Completions of Dedekind prime rings as second endomorphism rings...

B. T. Y. Kwee, On generalized translated quasi-Cesàro summability ...

Yves A. Lequain, Differential simplicity and complete integral closure

741

Mordechai Lewin, On nonnegative matrices.

753

Kevin Mor McCrimmon, Speciality of quadratic Jordan algebras ...

Hussain Sayid Nur, Singular perturbations of differential equations in abstract spaces .....................................

D. K. Oates, A non-compact Krein-Milman theorem .

Lavon Barry Page, Operators that commute with a unilateral shift on an invariant subspace...

Helga Schirmer, Properties of fixed point sets on dendrites.

Saharon Shelah, On the number of non-almost isomorphic models of $T$ in a

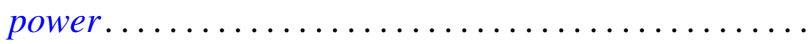

Robert Moffatt Stephenson Jr., Minimal first countable Hausdorff spaces....

Masamichi Takesaki, The quotient algebra of a finite von Neumann algebra 